\title{
Field-Assisted and Thermionic Contributions to Conductance in $\mathrm{SnO}_{2}$ Thick-Films
}

\author{
C. Malagù, ${ }^{1}$ M. C. Carotta, ${ }^{1}$ G. Martinelli, ${ }^{1}$ M. A. Ponce, ${ }^{2}$ M. S. Castro, ${ }^{2}$ and C. M. Aldao ${ }^{2}$ \\ ${ }^{1}$ Department of Physics, University of Ferrara, Via Saragat 1/C, 44100 Ferrara, Italy \\ ${ }^{2}$ Institute of Materials Science and Technology (INTEMA), National University of Mar del Plata, CONICET, Juan B. Justo 4302, \\ B7608FDQ Mar del Plata, Argentina \\ Correspondence should be addressed to C. Malagù, malagu@fe.infn.it
}

Received 27 January 2009; Revised 27 May 2009; Accepted 23 June 2009

Recommended by Giorgio Sberveglieri

\begin{abstract}
A deep analysis of conductance in nanostructured $\mathrm{SnO}_{2}$ thick films has been performed. A model for field-assisted thermionic barrier crossing is being proposed to explain the film conductivity. The model has been applied to explain the behavior of resistance in vacuum of two sets of nanostructured thick-films with grains having two well-distinct characteristic radii $(R=25 \mathrm{~nm}$ and $R=125 \mathrm{~nm}$ ). In the first case the grain radius is shorter than the depletion region width, a limit at which overlapping of barriers takes place, and in the second case it is longer. The behavior of resistance in the presence of dry air has been explained through the mechanism of barrier modulation through gas chemisorption.
\end{abstract}

Copyright $(\odot) 2009$ C. Malagù et al. This is an open access article distributed under the Creative Commons Attribution License, which permits unrestricted use, distribution, and reproduction in any medium, provided the original work is properly cited.

\section{Introduction}

In the last two decades, gas sensors based on $\mathrm{SnO}_{2}$ thick films became the dominant solid state devices for the gas detection in domestic, commercial, and industrial alarms $[1,2]$. It is widely accepted that, when oxygen chemisorbs, electrons transfer from the bulk to the surface of the grain modifying the barriers at the grain boundaries [3]. In particular, after oxygen chemisorption, the barrier height, $V_{s}$, and the depletion width, $\Lambda$, become larger and, as a consequence, the sample resistance increases. Different factors (such as type of defects, morphology, and additives) contribute to the electrical response of the gas sensor $[4,5]$.

The film resistivity (or conductivity) is broadly used to characterize a sensor, and the widely acknowledged phenomenological equation $[2,6,7]$ is

$$
\rho=\rho_{o} \operatorname{Exp}\left(\frac{e V_{s}}{k T}\right)
$$

where $\rho_{o}$ is the bulk resistivity, $k$ the Boltzmann constant, and $T$ the absolute temperature.

In this work, the resistance of undoped $\mathrm{SnO}_{2}$ thickfilm gas sensors is analyzed as a function of temperature in vacuum and in dry air atmosphere. In order to explain the results, thermionic and tunneling contributions to the electrical conduction have been considered.

\section{Experimental}

Commercial high-purity $\mathrm{SnO}_{2}$ (Aldrich, medium particle size $0.4 \mu \mathrm{m}$ ) was ground until a medium particle size of $0.1 \mu \mathrm{m}$ (labeled powders $\mathrm{P} 1$ ). A calcination process carried out at $1100^{\circ} \mathrm{C}$ for 2 hours led to powders with larger particle size (labeled powders $\mathrm{P} 2$ ). Then, a paste was prepared with an organic binder (glycerol) and the powders P1 and P2. The used solid/organic binder ratio was 1/2. No dopants were added.

Thick, porous film samples were made by painting onto insulating alumina substrate on which electrodes with an interdigit shape have been deposited by sputtering. Finally, samples were thermally treated for 2 hours in air at $500^{\circ} \mathrm{C}$. Samples were labeled S1 (small particle size) and S2 (large particle size). The thickness of the films was measured with a Surtronic $3^{+}$(Taylor Hobson) profilometer with a diamond stylus (radius: $1 \mu \mathrm{m}$ ). To image the tin oxide surfaces a JEOL JSM 6700F SEM was employed. The X-ray data were collected with a Siemens D-500 difractometer using $\mathrm{CuK} \alpha$ radiation. 
Dynamical resistance curves were measured while changing the vacuum into air flux and, after having reached quasisaturation, changing the dry air flux back into vacuum $\left(10^{-4} \mathrm{mmHg}\right)$. In temperature cycling experiments, resistance was measured while raising and then decreasing the temperature from room temperature up to $420^{\circ} \mathrm{C}$ at a rate of $\sim 2^{\circ} \mathrm{C} / \mathrm{min}$ with the sample kept in air $(40 \mathrm{mmHg})$.

\section{Modelling}

To model the resistance of the two sets of sensors, a fieldassisted mechanism for barrier crossing is proposed (see [8]). We applied the WKB approximation to a double parabolic barrier of the following form:

$$
V(r)= \begin{cases}\frac{V_{s}}{\Lambda^{2}}(r+\Lambda)^{2}, & -\Lambda \leq r \leq 0, \\ \frac{V_{s}}{\Lambda^{2}}(r-\Lambda)^{2}, & 0 \leq r \leq \Lambda\end{cases}
$$

The tunnelling and thermionic contributions to the current density, with respect to the flat band case, were calculated following the classical work of Crowell and Rideout for a single parabolic barrier [9]. The whole calculation resulted in a factor two in the exponent of the tunneling part of $[9$, equation (13)]. Thus, we found for the tunneling contribution, here called $I_{1}$, and for the thermionic one, here called $I_{2}$, respectively,

$$
\begin{gathered}
I_{1}=\frac{e V_{s}}{k T} \int_{0}^{1} \operatorname{Exp}\left\{-\frac{e V_{s}}{k T}\left[\alpha+\frac{2 k T y(\alpha)}{E_{00}}\right]\right\} d \alpha, \\
I_{2}=\operatorname{Exp}\left(-\frac{e V_{s}}{k T}\right),
\end{gathered}
$$

where

$$
\begin{gathered}
y(\alpha)=(1-\alpha)^{0.5}-\alpha \operatorname{Ln}\left[\frac{1+(1-\alpha)^{0.5}}{\alpha^{0.5}}\right], \\
\alpha=\frac{E}{e V_{s}} \\
E_{00}=\frac{e h}{4 \pi}\left(\frac{N_{d}}{m * \varepsilon}\right)^{1 / 2},
\end{gathered}
$$

with $E$ being the energy of an electron in the conduction band. From numerical solutions of (3) it turns out that the relative tunneling emission with respect to the thermionic one $\left(I_{1} / I_{2}\right)$ is an increasing function of $E_{00} / k T$ ratio. For a given material, $E_{00}$ is fixed; thus the tunneling contribution is more effective at lower temperatures. Moreover the solution of (3) at a fixed temperature shows, as discussed in [8] (our factor two does not alter the trend), that the lower the barrier the more important the relative thermionic contribution with respect to the field assisted one. This phenomenon gives an explanation to the experimental evidence presented below.

\section{Results and Discussion}

In Figures 1(a) and 1(b), SEM micrographs of the samples S1 and S2 are shown. Both samples present homogeneous

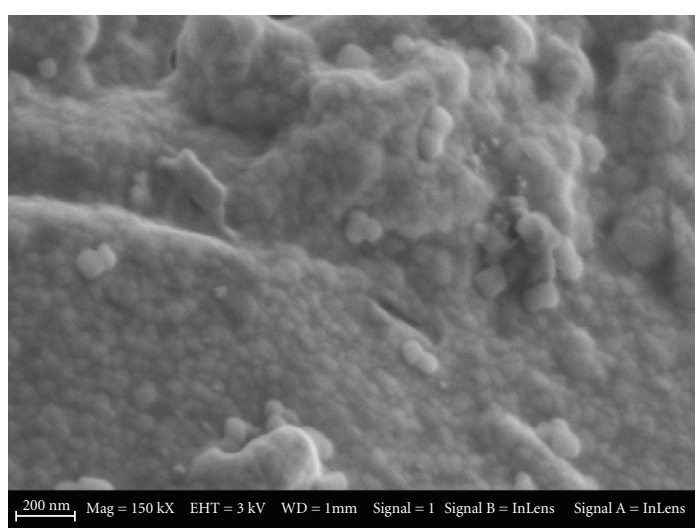

(a)

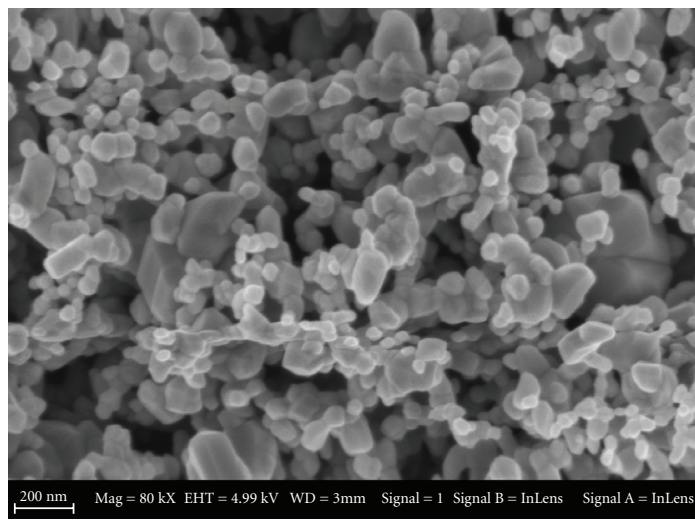

(b)

FIGURE 1: (a) SEM micrograph for a sample labeled $\mathrm{S} 1(R<\Lambda)$. (b) SEM micrograph for a sample labeled S2 $(R>\Lambda)$.

microstructures. From SEM micrographs a significant difference in the microstructures of S1 and S2 is observed. The samples showed the presence of agglomerates, but samples made with larger particle size (S2) showed a highly porous microstructure with the presence of some cracks (not shown in this figure). The average particle radius of samples labeled S1 was determined to be $25 \mathrm{~nm}$, while for samples labeled S2 it was determined to be $125 \mathrm{~nm}$. The mean thickness of the films determined by the profilometer was $100 \mu \mathrm{m}$ for samples S1 and $440 \mu \mathrm{m}$ for samples S2.

Since SEM imager for sample S1 is not clear enough to provide reliable grain size determination, we have performed X-Ray Diffraction (XRD) on this sample for comparison. From XRD, the film is found to be composed of $\mathrm{SnO}_{2}$ with a high degree of crystalline. In Figure 2, the peak corresponding to the (211) plane is showed. The grain sizes of the samples were calculated following Scherrer's law [10]:

$$
t=\frac{0,9 \cdot \lambda}{B \cdot \cos \left(\theta_{B}\right)},
$$

where $t$ is the $\mathrm{SnO}_{2}$ particle size; $\lambda$ is the wavelength (Co = $1.788 \AA$ ); $B$ is the angular width (in terms of $2 \theta_{B}$ ) measured in radians that corresponds to the full width at half-maximum; $\theta_{B}$ is the value of the angle of the peak of the highest intensity. Through this equation, a particle size, $t$, of $41 \mathrm{~nm}$ 


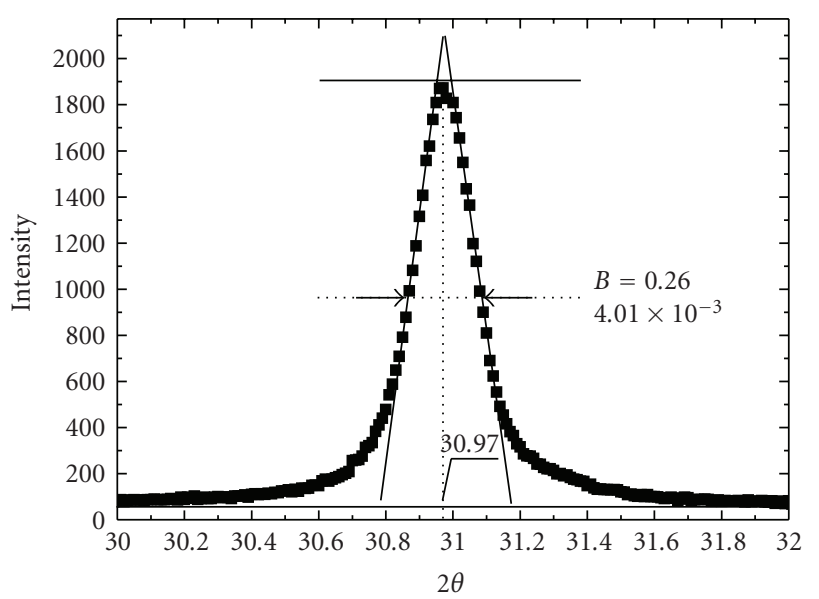

- Sample 1

FIgURE 2: XRD of sample 1 to compare with SEM measurement.

was calculated for sample S1. This result is in agreement with that found using SEM.

Figure 3 shows the electrical response (resistance versus time) of the sample with smaller particle size after changing the vacuum $\left(10^{-2} \mathrm{mmHg}\right)$ into air flux $(t=0)$ and back into vacuum. In Figure 3, curve A-experiment carried out at $280^{\circ} \mathrm{C}$-after a quick increasing due to air exposure, the electrical resistance is almost constant over a lapse of 700 seconds. On the other hand, at the temperature of $420^{\circ} \mathrm{C}$ (Figure 3, curve B) a diminution in the resistance after a quick increase is observed. Figure 4 shows electrical responses (resistance versus time) of the sample S2 after changing the vacuum into air flux $(t=0)$ and back into vacuum. Curve A shows an electrical response similar to those observed in sample S1 at the low temperature (Figure 3, curve A). When the temperature is increased at values close to $420^{\circ} \mathrm{C}$, a slow decreasing in the resistance with exposure time was registered (Figure 4, curve B). This behavior is similar to that observed at the high temperature in sample $\mathrm{S} 1$ for the longest exposition times. However, due to the greater thickness in sample S2, a slower electrical response with respect to $\mathrm{S} 1$ in the studied temperature range is detected.

Resistance versus time curves can be understood by considering that intergranular potential barriers are responsible for the observed electrical response [11]. The rapid increase of the resistance, when samples are exposed to air, indicates that equilibrium at the surface is quickly reached. The interaction of oxygen with grain surfaces produces the transfer of electrons from the bulk to the surface. From this process, $V_{s}$ and $\Lambda$ become larger and, as a consequence, the sample resistance increases. The long term changes in the resistance observed after a quick initial change in the atmosphere can be explained in terms of oxygen in- and outdiffusion [12] that reflects in a slow change of the doping level, $N_{d}$.

Samples with larger particle size (S2) showed a relatively slower response than samples with smaller particle size (S1). This result could be explained by considering that samples

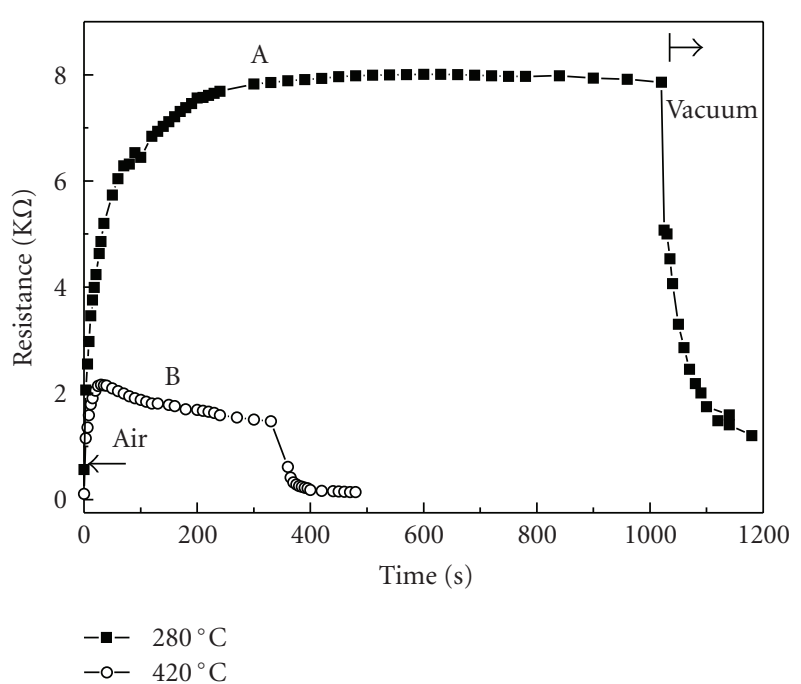

Figure 3: Dynamical response (resistance versus time) of S1 after changing the vacuum into air flux $(t=0)$ and back into vacuum. (a) $\mathrm{T}=280^{\circ} \mathrm{C}$. (b) $\mathrm{T}=420^{\circ} \mathrm{C}$.

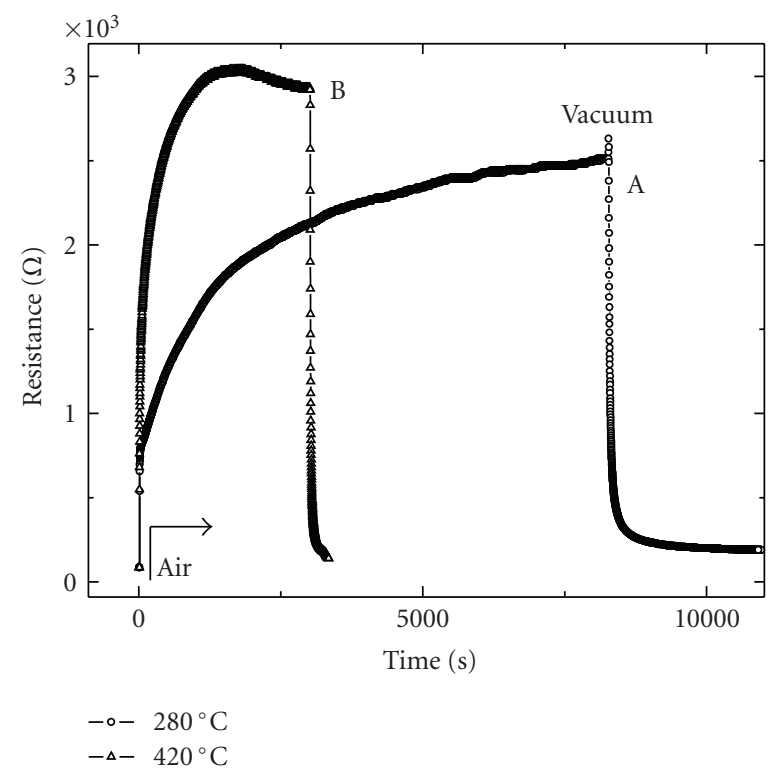

Figure 4: Dynamical response (resistance versus time) of S2 after changing the vacuum into air flux $(t=0)$ and back into vacuum. (a) $\mathrm{T}=280^{\circ} \mathrm{C}$. (b) $\mathrm{T}=420^{\circ} \mathrm{C}$.

S2 are thicker than S1. It has long been suggested that the response time of a semiconductor gas sensor could be related to the reactivity and diffusion of gas molecules inside gas sensing layers $[13,14]$.

Regarding the behaviour of resistance in vacuum a more subtle analysis is required. The values of resistance in vacuum for the samples S1 and S2 are reported in Table 1 at the temperatures of 280 and $420^{\circ} \mathrm{C}$, respectively.

Samples S2 show a lower resistance than samples S1 at both temperatures due to the lower number of grains. But it is worth noticing that, whereas at the high temperature the value of the resistance is comparable to that of the smaller 


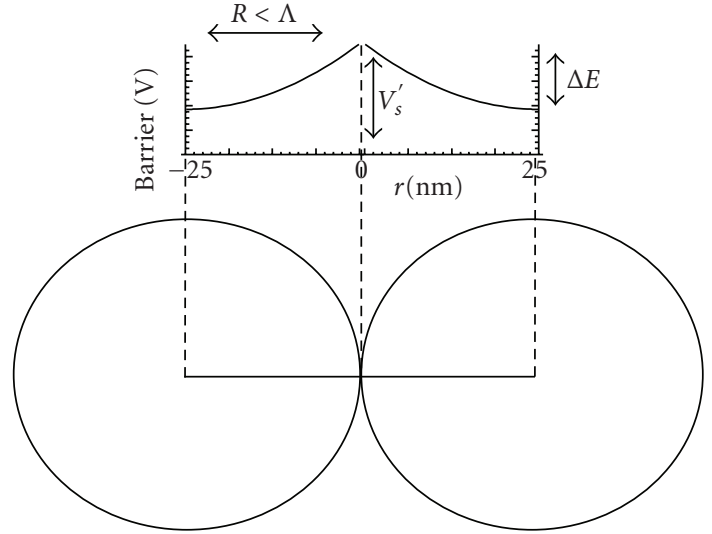

FIgURE 5: $R<\Lambda$, the potential is not zero at the centre of the grain. The potential barrier that electrons have to cross is $\Delta E<e V_{s}^{\prime}$. $V_{s}^{\prime}$ is the distance between the conduction band at surface and the Fermi level; it holds $V_{s}^{\prime}<V_{s}[13]$.

TABLE 1: Average resistance values in vacuum of samples S1 and S2 at the two operating temperatures.

\begin{tabular}{lcc}
\hline & $\mathrm{S} 1(R=25 \mathrm{~nm})$ & $\mathrm{S} 2(R=125 \mathrm{~nm})$ \\
\hline $280^{\circ} \mathrm{C}$ & $R=0.565 \mathrm{k} \Omega$ & $R=0.105 \mathrm{k} \Omega$ \\
$420^{\circ} \mathrm{C}$ & $R=0.110 \mathrm{k} \Omega$ & $R=0.085 \mathrm{k} \Omega$ \\
\hline
\end{tabular}

grained sample, at the low temperature the resistance of $S 1$ is five times larger than that of S2. The case of grains with $R<\Lambda$ (S1) is sketched in Figure 5. In a previous work [15] we have demonstrated that the barrier height in vacuum, which we will call here $V_{s}^{\prime}$, of $\mathrm{SnO}_{2}$ grains with $R<\Lambda$ is expected to be about $10 \%$ lower than that of grains with $R>\Lambda$ because of the decrease of surface state density.

It must be observed that the density of electrons decreases exponentially with the distance of $E_{c}$ from $E_{F}$ in the same manner as the thermionic contribution increases due to the lower barrier. As explained in [8, equation (7)], if we call $\Delta E$ the barrier height and $e V_{s}^{\prime}$ the distance between $E_{c}$ and $E_{F}$, then the Fermi level is $e V_{s}^{\prime}-\Delta E$ below the bottom of the conduction band. The carrier density is then reduced by a factor $\operatorname{Exp}\left[-\left(e V_{s}^{\prime}-\Delta E\right) / k T\right]$. Consequently the total thermionic contribution to conductance is proportional to $\operatorname{Exp}\left[-\left(e V_{s}^{\prime}-\Delta E\right) / k T\right] \times \operatorname{Exp}(-\Delta E / k T)$, where the first term is the change in carrier density and the second one is the probability of barrier crossing. This product gives exactly

$$
\operatorname{Exp}\left[\frac{-e V_{s}^{\prime}}{k T}\right]=\operatorname{Exp}\left[-\frac{E_{c}-E_{F}}{k T}\right] \text {. }
$$

Therefore, if we do not invoke tunnelling, it is necessary for a semiconductor with lower distance between $E_{c}$ and $E_{F}$ to exhibit higher conductivity, which is contradicted by experimental evidence.

If the mechanism of barrier crossing were purely thermionic, the smaller grains would show less resistivity at every temperature, being the resistivity an exponential function of the barrier height; see (6). At the higher temperature, the number of electrical active grains, of the samples S2, competes with its higher resistivity making the resistance of the two samples almost equal, $R(\mathrm{~S} 1)=1.3 R(\mathrm{~S} 2)$. Instead, at $280^{\circ} \mathrm{C}$ where the tunneling contribution $\left(I_{1}\right)$ is dominant with respect to the thermionic $\left(I_{2}\right)$ because at lower temperature $E_{00} \cong k T$, the effect of grain size is crucial. In the case of small grains, the actual barrier to be crossed, $\triangle E$, is much lower than that of the larger grains, and, in the case of lower barrier, the relative tunneling contribution is reduced with respect to the thermionic one. In fact, at $R=25 \mathrm{~nm}$ the overlapping of potential barriers takes place, see Figure 5, and $\Delta E<V_{s}^{\prime}<V_{s}$. Therefore a larger resistance for smaller grains is to be expected at the lower temperature. If instead the phenomenon were purely determined by thermionic emission, the barrier lowering of small grains could not produce different effects at different temperatures, the effect being only that of reducing the resistance of the small grains with respect to that of large ones. It would not then be possible to explain why $R(\mathrm{~S} 1)=$ $5.4 R(\mathrm{~S} 2)$ at $280^{\circ} \mathrm{C}$.

\section{Conclusions}

The resistance behavior of the two samples in oxygen has been explained in terms of barrier variations due to surface reactions. In the case of vacuum, to explain the greater resistance (Table 1) of small grains with respect to larger ones, in spite of the fact that the barrier of the latter is higher, it is necessary to invoke tunneling. It turns out that the relative tunneling contribution in the case of small grains is smaller because intergranular barriers are lower as a consequence of the lift of the potential at the centre of the grain.

Therefore, if the sensors are working at temperatures at which the tunneling crossing is dominant with respect to thermionic, it is possible that the decrease of the former contribution results in a very big increase in resistance for samples with a grain radius smaller than depletion width. This evidence could not be taken into account by bare thermionic emission.

\section{Acknowledgment}

This work has been possible thanks to the project of Scientific Cooperation between CNR (Italy) and CONICET (Argentina).

\section{References}

[1] W. Göpel and K. D. Schierbaum, " $\mathrm{SnO}_{2}$ sensors: current status and future prospects," Sensors and Actuators B, vol. 26, no. 1-3, pp. 1-12, 1995.

[2] N. Barsan and U. Weimar, "Conduction model of metal oxide gas sensors," Journal of Electroceramics, vol. 7, no. 3, pp. 143$167,2001$.

[3] G. Gaggiotti, A. Galdikas, S. Kaciulis, G. Mattogno, and A. Setkus, "Surface chemistry of tin oxide based gas sensors," Journal of Applied Physics, vol. 76, no. 8, pp. 4467-4471, 1994.

[4] S. Capone, P. Siciliano, F. Quaranta, R. Rella, M. Epifani, and L. Vasanelli, "Moisture influence and geometry effect of $\mathrm{Au}$ and 
Pt electrodes on $\mathrm{CO}$ sensing response of $\mathrm{SnO}_{2}$ microsensors based on sol-gel thin film," Sensors and Actuators B, vol. 77, no. 1-2, pp. 503-511, 2001.

[5] C. Xu, J. Tamaki, N. Miura, and N. Yamazoe, "Grain size effects on gas sensitivity of porous $\mathrm{SnO}_{2}$-based elements," Sensors and Actuators B, vol. 3, no. 2, pp. 147-155, 1991.

[6] J. W. Orton and M. J. Powell, "The Hall effect in polycrystalline and powdered semiconductors," Reports on Progress in Physics, vol. 43, no. 11, pp. 1263-1307, 1980.

[7] P. Romppainen and V. Lantto, "The effect of microstructure on the height of potential energy barriers in porous tin dioxide gas sensors," Journal of Applied Physics, vol. 63, no. 10, pp. 5159-5165, 1988.

[8] C. Malagú, G. Martinelli, M. A. Ponce, and C. M. Aldao, "Unpinning of the Fermi level and tunneling in metal oxide semiconductors," Applied Physics Letters, vol. 92, no. 16, Article ID 162104, 3 pages, 2008.

[9] C. R. Crowell and V. L. Rideout, "Normalized thermionic-field (T-F) emission in metal-semiconductor (Schottky) barriers," Solid State Electronics, vol. 12, no. 2, pp. 89-105, 1969.

[10] B. D. Cullity, "Elements of X-Ray Diffraction," AddisonWesley, Reading, Mass, USA, 1978.

[11] G. Blaustein, M. S. Castro, and C. M. Aldao, "Influence of frozen distributions of oxygen vacancies on tin oxide conductance," Sensors and Actuators B, vol. 55, no. 1, pp. 3337, 1999.

[12] M. A. Ponce, C. Malagu, M. C. Carotta, G. Martinelli, and C. M. Aldao, "Gas indiffusion contribution to impedance in tin oxide thick films," Journal of Applied Physics, vol. 104, no. 5, Article ID 054907, 5 pages, 2008.

[13] G. Sakai, N. S. Baik, N. Miura, and N. Yamazoe, "Gas sensing properties of tin oxide thin films fabricated from hydrothermally treated nanoparticles: dependence of $\mathrm{CO}$ and $\mathrm{H}_{2}$ response on film thickness," Sensors and Actuators B, vol. 77, no. 1-2, pp. 116-121, 2001.

[14] M. A. Ponce, C. M. Aldao, and M.S . Castro, "Influence of particle size on the conductance of $\mathrm{SnO}_{2}$ thick films," Journal of the European Ceramic Society, vol. 23, no. 12, pp. 2105-2111, 2003.

[15] T. G. G. Maffers, G. T. Owen, C. Malagú, et al., "Direct evidence of the dependence of surface state density on the size of $\mathrm{SnO}_{2}$ nanoparticles observed by scanning tunnelling spectroscopy," Surface Science, vol. 550, no. 1-3, pp. 21-25, 2004. 

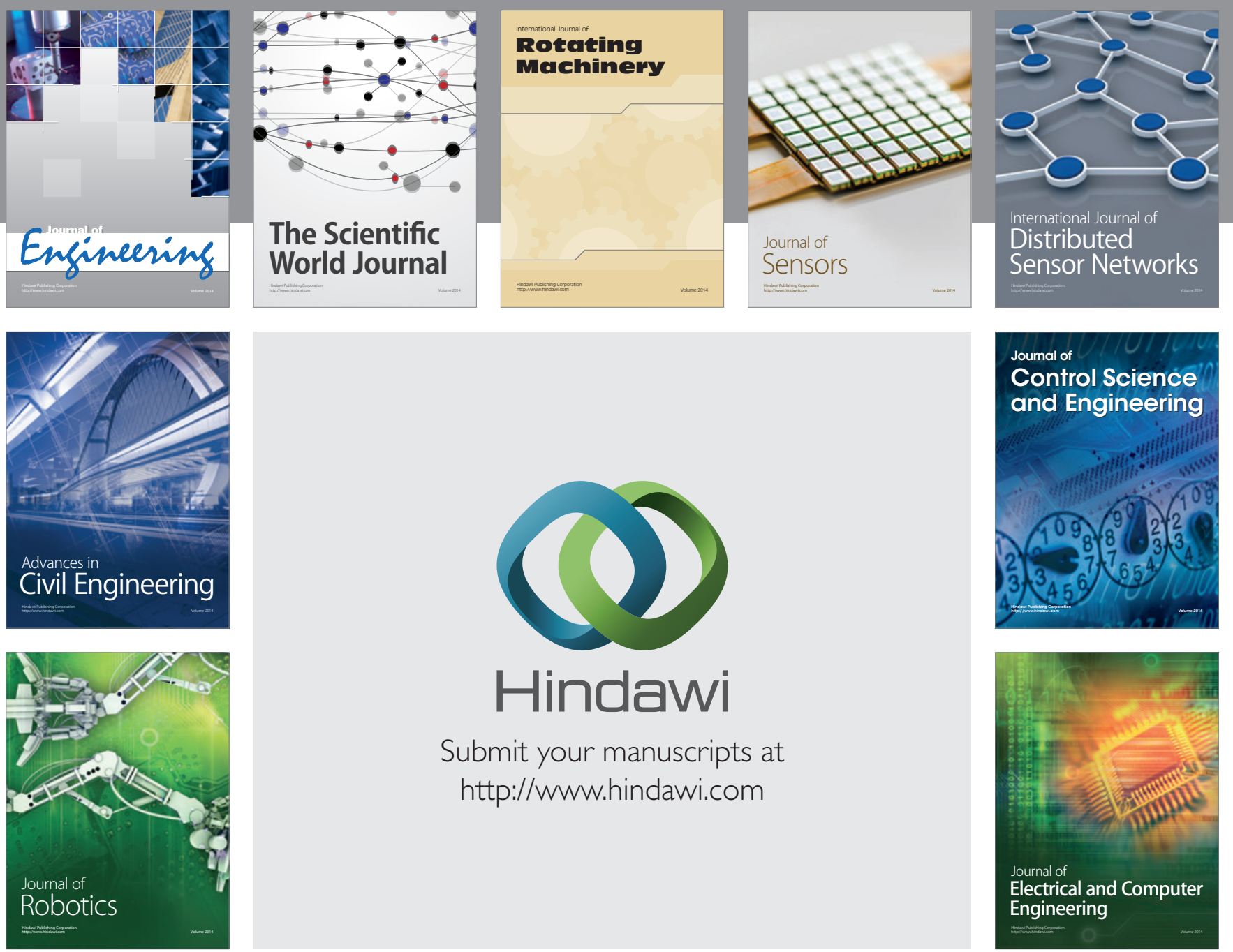

Submit your manuscripts at

http://www.hindawi.com
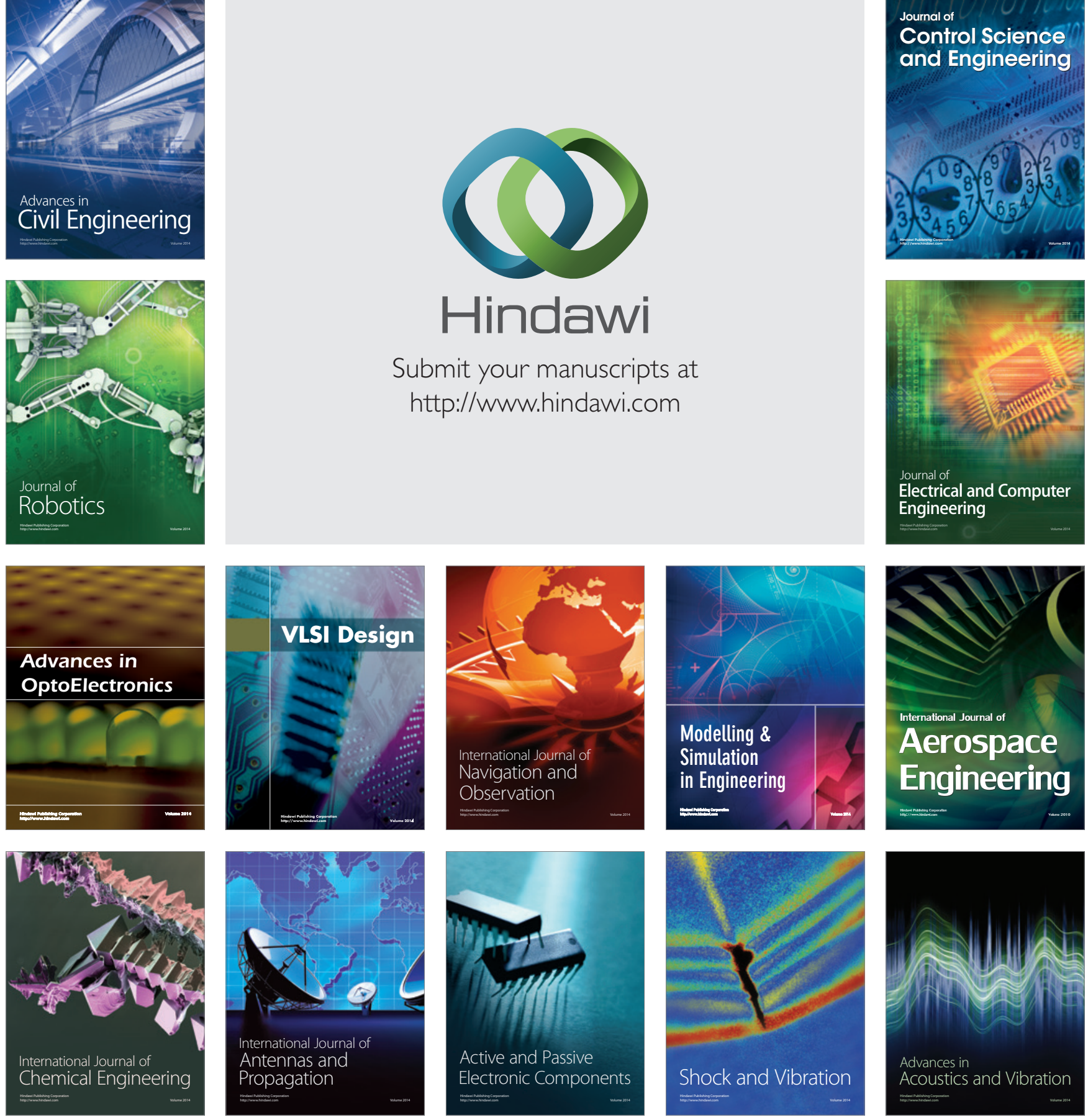